\title{
Sonic Hedgehog Improved Endothelial Cell Dysfunction and Attenuated The Development of Atherosclerosis In Mice
}

Huizhen Yu

Fujian Provincial Hospital

Huashan Huang

Fujian Provincial Hospital

Yan Xue

Fujian Provincial Hospital

Lichao Lin

Fujian Medical University

Wenfei Zeng

Fujian Medical University

Pengli Zhu ( $\nabla$ zhupengli7755@163.com )

Fujian Provincial Hospital https://orcid.org/0000-0003-1229-167X

\section{Research Article}

Keywords: Sonic hedgehog, Atherosclerosis, Endothelial dysfunction, Inflammation

Posted Date: December 1st, 2021

DOI: https://doi.org/10.21203/rs.3.rs-1043515/v1

License: (c) (i) This work is licensed under a Creative Commons Attribution 4.0 International License. Read Full License 


\section{Abstract}

Sonic hedgehog (Shh), an evolutionally-highly conserved morphological factor whose maturation, transportation and function were closely related to cholesterol. Shh played an important role in maintaining adult coronary vasculature homeostasis. It not only induced angiogenesis to improve myocardial infarction but also inhibited ox-LDL induced endothelial apoptosis. However, the role of Shh in endothelial cell injury have not been fully elucidated. Here, we shown Shh induced nitric oxide (NO) release and endothelial nitric oxide synthase (eNOS) synthesis, which improved endothelial cell dysfunction and inhibited atherosclerotic plaque. In vivo, Shh reduced the plaque lesion in high fat diet (HFD) induced $\mathrm{ApoE}^{-/-}$mice. In endothelial cell, Shh improved NO and eNOS mRNA expression and inhibited intercellular adhesion molecule 1 (ICAM-1) and vascular cell adhesion molecule 1 (VCAM-1) mRNA expression. In contrast, knockdown of Shh inhibited eNOS and NO level and induced ICAM-1 and VCAM-1. In conclusion, we found that Shh has anti-inflammation and improved endothelial cell injury consequently attenuated the development of atherosclerosis.

\section{Introduction}

Endothelial cell dysfunction was regarded as the crucial and early change in the life history of atherosclerotic lesion and was a key factor to the underlying pathobiology of atherosclerotic cardiovascular disease (ASCVD)[1]. Changes in vascular endothelium, broadly speaking, included a range of modulations in functional phenotypes, vascular tension, redox balance and inflammation, which provided a vital link to atherosclerotic lesion initiation and progression[2]. Multiple factors such as hyperlipidemia, hypertension, hyperglycemia and smoking derived circulating low density lipoprotein $(\mathrm{LDL})$ accumulation in the arterial lesion-prone zone and LDL oxidation modification into oxidized low density lipoprotein (ox-LDL), which resulted in augment of reactive oxygen species (ROS), inhibition of the endothelial nitric oxide synthase (eNOS) synthesis and nitric oxide (NO) release, subsequently, elevated expression of cell surface adhesion factors including intercellular adhesion molecule 1 (ICAM-1) and vascular cell adhesion molecule 1 (VCAM-1), recruiting of circulating monocytes to the intima, induced a complex process of endothelial cell dysfunction and launched pathogenic occlusion[3]. Focusing on the causes and courses of endothelial cell dysfunction and inhibiting them have been an important therapeutic target for ASCVD.

Sonic hedgehog (Shh) was a highly evolutionarily conserved morphogen which could shape into concentration gradients to provide cells location information and determine cell fates[4]. Shh morphogen served as an important role in the development and homeostasis maintenance of blood vessels in embryo and postnatal and abnormal expression of Shh was associated with various birth defects and tumors. As previously studies, Shh established their morphogen gradients nothing but covalently modified by cholesterol and palmitoyl groups[5]. Shh lacking cholesterol resulted in the change of subcellular distribution and seriously disrupted the organization structure[6]. Functional Shh contained cholesterol and palmitoyl hydrophobic moieties which was manifestly incompatible with long-distance functions. Drosophila hedgehog could be carried by the insect lipophorin which strongly resemble 
mammalian LDL, and lipophorin-bound hedgehog was capacity to transport to target cells[7]. A set of studies demonstrated that ox-LDL was strongly associated with progression of atherosclerosis, thusly, further investigating the relationship between ox-LDL and Shh is with large unmet medical needs.

In this study, we addressed the potential role of Shh in ox-LDL induced endothelial cell dysfunction and atherosclerosis. In vivo, Shh attenuated the action of high fat diet (HFD) on the atherosclerosis plaque area in $\mathrm{ApoE}^{-/-}$mice. And also, Shh improved NO and eNOS expression. In vitro, upon ox-LDL treatment, eNOS and NO were downregulated and ROSIICAM-1 and VCAM-1 expression were upregulated and in human umbilical vein endothelial cells (HUVECs). Overexpression of Shh by recombinant Shh protein or plasmid encoding Shh gene induced eNOS and NO expression, and on the other hand, ameliorated ROS and ICAM-1, VCAM-1 mRNA expression. In contrast, silencing of Shh by siRNA, accelerated ox-LDLinduced endothelial cell dysfunction and could be rescued partly by recombinant Shh protein. Altogether, our data briefly indicated that Shh could improve endothelial cell dysfunction.

\section{Materials And Methods}

\subsection{Chemicals and regents}

Human oxidized LDL was purchased from Yiyuan biotech (Guangzhou, China). The rabbit Shh antibody was purchased from Cell Signaling Technology (Danvers, USA). Oil Red 0 solution, $0.5 \%$ in isopropanol, was purchased from Sigma Aldrich (St. Louis, MO, USA). Reactive Oxygen Species Assay kit (ROS Assay Kit) was purchased from Beyotime Biotechnology (Shanghai, China). Nitric Oxide Assay kit (NO Assay Kit) was purchased from Beyotime Biotechnology (Shanghai, China).

\subsection{Animal Model}

Male $\mathrm{ApoE}^{-/-}$mice aged 8 weeks old on a C57BL/6 background were obtained from Model Animal Research Center of Nanjing University, China. All mice were kept on a standard lighting (12 h light/12 h dark cycle), constant temperature $\left(22-24^{\circ} \mathrm{C}\right)$, and humidity (50-60\%). Experiment mice were given a High Fat Diet (HFD). We randomly divided the mice into three groups for intravenous injection treatment $(n=20$ in each group): Lv-Shh group (lentivirus at a dose of $2^{\star} 10^{7} \mathrm{TU} /$ mouse), Sh-Shh group (lentivirus at a dose of $2^{\star} 10^{7} \mathrm{TU} /$ mouse), and negative group. At the end of 12 weeks, all the mice were euthanized for subsequent study. The animal care and procedures were complied with the guidelines of animal welfare.

\subsection{Tissue preparation}

The whole aortas and hearts were rapidly removed and washed in PBS. In en face oil red 0 staining, the adventitia of aortas was identified by oil red $\mathrm{O}$. In en face oil red $\mathrm{O}$ staining, the adventitia of aortas was stripped at first, then the aortic arches were dissected.

\subsection{Cell culture}


HUVECs were obtained from human umbilical cord veins treated with a $0.25 \%$ trypsin solution following previously published methods. Cells were cultured in Endothelial Cell Medium (ScienCell Research Laboratories, Inc.) consisting of basal medium, 5\% FBS, 1\% EC growth supplement and 1\% penicillin/streptomycin solution in a humidified incubator at $37^{\circ} \mathrm{C}$ in a $5 \% \mathrm{CO}_{2}$ atmosphere. Cells were used between passages 3 to 5 .

\subsection{Plasmid transfection}

Commercialized plasmid encoding the human Shh gene (phShh) were synthesized by General Biosystems Co., Ltd. PhShh were constructed by inserting a 1425 bp Shh gene into the plasmid pcDNA3.1/Hygro(+). HUVECs were transfected with $2 \mu \mathrm{g}$ phShh using Lipofectamine 3000 transfection reagent (Invitrogen, Thermo Fisher Scientific, Inc.) and then cultured for $48 \mathrm{~h}$ before subsequent experiments.

\subsection{RNA isolation and real-time PCR assay}

Total RNA was extracted using TRIzol regent (Invitrogen, Thermo Fisher Scientific, Inc.). The total RNA (1000 ng) was subjected to a reverse transcription reaction with the PrimeScriot ${ }^{\mathrm{TM}}$ room temperature (RT) Reagent Kit (Takara, Japan). Real-PCR was carried out using Sybr Premix Ex Taq ${ }^{\mathrm{TM}}$ II (Takara, Japan).

Table 1

The primer sequences for the qRT-PCR

\begin{tabular}{|c|c|c|}
\hline Species & Gene Symbol & Primer Sequences \\
\hline Homo Sapien & eNOS FW & 5'-CGAGATATCTTCAGTCCCAAGC-3' \\
\hline Homo Sapien & eNOS RV & 5'-GTGGATTTGCTGCTCTCTAGG-3' \\
\hline Homo Sapien & ICAM-1 FW & 5'-TTGGGCATAGAGACCCCGTT-3' \\
\hline Homo Sapien & ICAM-1 RV & 5'-GCACATTGCTCAGTTCATACACC-3' \\
\hline Homo Sapien & VCAM-1 FW & 5'-GGGAAGATGGTCGTGATCCTT-3' \\
\hline Homo Sapien & VCAM-1 RV & 5'-TCTGGGGTGGTCTCGATTTTA-3' \\
\hline Homo Sapien & $\beta$-actin FW & 5'-AGAAGGCTGGGGCTCATTTG-3' \\
\hline Homo Sapien & $\beta$-actin RV & 5'-AGGGGCCATCCACAGTCTTC-3' \\
\hline Mouse & eNOS FW & 5'-GAAGGTCTTCTACGTGATCGAG-3' \\
\hline Mouse & eNOS RV & 5'-TTCAGCCACCACGTACAC-3' \\
\hline Mouse & $\beta$-actin FW & 5'-CTACCTCATGAAGATCCTGACC-3' \\
\hline Mouse & $\beta$-actin RV & 5'-CACAGCTTCTCTTTGATGTCAC-3' \\
\hline
\end{tabular}

\subsection{Statistical analysis}


All statistical analysis was performed using GraphPad Prism 8.0. The data were presented as the mean \pm standard deviation (SD), and one-way ANOVA was used to perform statistical comparisons followed by Tukey's post hoc test. $\mathrm{P}<0.05$ was considered to indicate a statistically significant difference.

\section{Results}

\subsection{Effect of Shh in $\mathrm{ApoE}^{-/-}$mice in the atherosclerosis}

To test the effect of Shh in HFD induced $\mathrm{ApoE}^{-/-}$mice, we operated by intravenous injection lentivirus including Lentivirus Shh and Shh shRNA by tail. Firstly, we verified that Shh gene transfer was successfully in aortic tissue (Figure 1A). Furthermore, we compared the mice artery plaque area after 12 weeks. As shown in Figure 1B, HFD induced the formation of atherosclerotic plaque especially in aorta, carotid artery and abdominal aorta, and compared with the negative control, lentivirus Shh could reduce the area of plaque, in contrast, Shh shRNA increased the plaque. It was interesting to note there was no significant difference among the negative control group, lentivirus Shh group and Shh shRNA group in weight and cholesterol including T-CHO, TG, LDL-C and HDL-C (Figure 1C). Furthermore, to assess the Shh effect on endothelium function, we measured the expression of NO in serum and eNOS mRNA and protein in aortic tissue. Lentivirus Shh induced the expression of NO in serum and eNOS mRNA and protein in aortic tissue, but Shh shRNA was inversive (Figure 1D). These results suggested that Shh inhibited endothelial cell dysfunction and attenuated the development of atherosclerosis in $\mathrm{ApoE}^{-/-}$mice.

Table 2

Effect of Shh in $\mathrm{ApoE}^{-/-}$mice in body weight and serum cholesterol

\begin{tabular}{|llllll|}
\hline Items & Chow & HFD & NC & Lv-Shh & Sh-Shh \\
\hline Weight $(\mathrm{g})$ & $28.98 \pm 1.01$ & $30.77 \pm 1.39$ & $30.84 \pm 1.52$ & $30.10 \pm 0.92$ & $31.62 \pm 1.38$ \\
\hline T-CHO $(\mathrm{mM})$ & $23.46 \pm 1.43$ & $30.58 \pm 1.71$ & $30.47 \pm 2.34$ & $28.83 \pm 1.49$ & $32.39 \pm 1.99$ \\
\hline $\mathrm{TG}(\mathrm{mM})$ & $1.71 \pm 0.20$ & $2.70 \pm 0.60$ & $2.67 \pm 0.59$ & $2.70 \pm 0.44$ & $2.87 \pm 0.54$ \\
\hline LDL-C(mM) & $3.79 \pm 0.45$ & $5.28 \pm 1.45$ & $5.43 \pm 0.75$ & $5.02 \pm 0.92$ & $5.94 \pm 0.89$ \\
\hline HDL-C(mM) & $1.48 \pm 0.13$ & $1.25 \pm 0.17$ & $1.26 \pm 0.28$ & $1.30 \pm 0.18$ & $1.22 \pm 0.12$ \\
\hline
\end{tabular}

T-CHO: Total cholesterol. TG: Triglyceride. LDL-C: Low density lipoprotein. HDL: High density lipoprotein. NC: Lentivirus control group. LV-Shh: lentivirus-Shh. Sh-Shh: Shh shRNA.

\subsection{Effect of Shh in endothelial cell dysfunction in Ox-LDL- Induced HUVECs}

It was reported that ox-LDL was an inducing factor in endothelial dysfunction[8]. In this study, we used HUVECs transfected with plasmid encoding Shh or pretreated with recombinant Shh protein followed by a $24 \mathrm{~h}$ co-incubation with ox-LDL $(50 \mu \mathrm{g} / \mathrm{mL})$ to observe the effect of Shh on endothelial dysfunction in vitro. Ox-LDL induced reactive oxygen in HUVECs, resulting in an increase in ROS level which is consistent 
with the previous results[9]. Furthermore, transfected with plasmid encoding Shh effectively suppressed the production of ROS (Figure 2A). Consistent with the results of plasmid encoding Shh gene, ROS level decreased in a dose-dependent manner after recombinant Shh protein administration (Figure 2B). In other hand, ox-LDL treatment induced significant downregulation of $\mathrm{NO}$ level, which was known as an effective endogenous vasodilator[10]. As shown in Figure 2C, plasmid encoding Shh gene could induced NO expression. Alike, compared with ox-LDL group, recombinant Shh protein motivated the releasing of serum NO level (Figure 2D). In keeping with NO expression, plasmid and recombinant protein of Shh induced eNOS mRNA expression (Figure 2E and F). These results shown that Shh improved endothelial cell dysfunction by downregulation of ROS and promoting the NO and eNOS expression.

\subsection{Effect of Shh on inflammation in ox-LDL-Induced HUVECS}

According to the protein-protein interaction in STRING network, we found that eNOS had relationship with ICAM-1 and VCAM-1 which members of cell adhesion molecules (Figure 3A). As former studies have proved cell adhesion molecules as inflammation factor accelerated endothelial cell dysfunction. We verified the effect of Shh on ICAM-1 and VCAM-1 in response to endothelial cell dysfunction. As shown in Figure 3B and C, ox-LDL treatment had a notable enhancement of ICAM-1 and VCAM-1 expression. Pretreatment with plasmid encoding Shh gene or recombinant Shh protin effectively suppressed the production of ICAM-1 and VCAM-1 mRNA level.

\subsection{Silencing Shh induced endothelial cell dysfunction and inflammation}

To further clarify Shh improved endothelial cell dysfunction and inhibited inflammatory response, we used siRNA to knockdown Shh. As shown in Figure 4A, siRNA 1, 2, 3 could interfere Shh mRNA expression and siRNA 2 was most remarkable, so we chosen siRNA2 for forward study. Transfected with Shh siRNA alone could induced the expression of ROS and ox-LDL in development the ROS level and could be weaken by recombinant Shh protein (Figure 4B). For another, ox-LDL treatment induced significant downregulation of NO level which was suppressed by recombinant Shh protein, and to the contrary, accelerated by knockdown of Shh (Figure 4C). Consistent with NO expression, siRNA inhibited eNOS mRNA expression (Figure 4D). Further, Shh siRNA could induced the expression of ICAM-1 and VCAM-1 and recombinant Shh protein could inhibit siRNA induced ICAM-1 and VCAM-1 expression to some extent (Figure 4E). Therefore, we demonstrated that silencing Shh could induce endothelial cell dysfunction and may rescue by recombinant Shh protein.

\section{Discussion}

Improving of endothelial cell dysfunction was a vital step in suppression the progression of atherosclerosis and preventing acute cardiovascular events, which bring out massive medical burden globally[11]. Therefore, it was of great significance to actively explore the molecular mechanism of atherosclerosis in the prevention and treatment of cardiovascular diseases and related complications. In 
preview study, we had identified that Shh expression was suppressed in ox-LDL-induced HUVECs and in addition, Shh could improve endothelial apoptosis. In this study, we found that Shh could promote NO and eNOS expression, leading to a significant suppression of atherosclerotic plaques in $\mathrm{ApoE}^{-/-}$mice. Consistent with these data, Shh could improve endothelial cell dysfunction by promoting expression of NO and eNOS and inhibiting expression of ROS and inflammation in ox-LDL-induced HUVECs. These results suggest that Shh was a promising therapeutic target for atherosclerosis.

Endothelial cell dysfunction is a major pathogenetic contributor of early-stage atherosclerotic lesions which is characterized by endothelial cells maladapted including reducing of eNOS and NO expression and inducing ROS expression, resulting in promoting endothelial cells activated and inflammation whereas vasoconstriction[12]. Under uncontrolled uptake of ox-LDL, impaired release of cholesterol, and excessive cholesterol esterification could result in accumulation of cholesterol esters stored as cytoplasmic lipid droplets and subsequently trigger endothelial dysfunction. Control of the homeostasis of endothelium has a critical importance in the pathogenesis of atherosclerosis, we observed that Shh exerted a protective effect by down-regulation ROS and promoted eNOS and NO releasing. Furthermore, we undertook an in vivo demonstrated that Shh could reduce the plaque lesion in HFD-diet ApoE ${ }^{-/-}$mice. These results provide strong evidence to support the notion that Shh contributed to a protective effect through increased eNOS and NO expression, as well as inhibited trafficking of cell surface molecules, thereby exerted anti-arteriosclerosis. Moreover, previous study has found that Shh could reduce the endothelial cell apoptosis associated with the NF-KB signaling. This remind us that this pathway may also be involved in the regulation of the endothelial cell dysfunction. Further work is needed before a definitive conclusion on this matter can be draw.

In recent years, scholars have found that Shh has neuroprotective effects on anti-oxidative stress and anti-apoptosis[13]. In the cortical neuron damage induced by $\mathrm{H}_{2} \mathrm{O}_{2}$, Shh could promote the expression of anti-apoptotic gene Bcl-2 and inhibit the expression of pro-apoptotic gene Bax, and simultaneously upregulate neurotrophic factor, vascular endothelial growth factor and brain-derived neurotrophic factor to protect cortical neurons from oxidative stress[14]. In addition, Shh increased the activities of superoxide dismutase and glutathione peroxidase, and up-regulates the expression of Bcl-2 protein, which improved the ability of autistic patients to defend against oxidative stress[15]. In previous study, in ox-LDL-induced HUVECs apoptosis model, we also found that Shh, as a protective protein, can inhibit the mitochondriamediated apoptosis. And further, in this study we found that Shh have anti-inflammation and endothelial relaxing.

In short, these data suggest that Shh may attenuate ox-LDL induced endothelial cell dysfunction through down-regulation of expression of ROS and and up-regulation of expression of NO. Thus, improving of Shh expression to reduce the injury of cells in early lesions could be important for reducing the number and size of cells in atherosclerotic lesions.

\section{Declarations}


Funding

This work was supported by the National Natural Science Foundation of China (No. 81873515), Natural

Science Foundation of Fujian (No. 2020J01123024) and high-level hospital foster grants from Fujian Provincial Hospital, Fujian province, China(No. 2020HSJJ03).

Competing Interests

The authors have no relevant financial or non-financial interests to disclose.

Author Contributions

The study conception and design were performed by Hui-zhen Yu, Hua-shan Huang and Peng-li Zhu. Material preparation, data collection and analysis were performed by Hui-zhen Yu, Hua-shan Huang, Xue Yan, Li-chao Lin, Wen-fei Zeng and Peng-li Zhu. The first draft of the manuscript was written by Hui-zhen Yu and Hua-shan Huang, and all authors commented on previous versions of the manuscript. All authors read and approved the final manuscript.

Data Availability

The datasets generated during and analysed are available from the corresponding author on reasonable request.

Ethics approval

This study was performed in line with the principles of the Declaration of Helsinki. Approval was granted by the Ethics Committee of Fujian Medical University (Date Mar. 2019 /No FJMU IACUC2019-0019).

\section{References}

1. Jensen HA, Mehta JL (2016) Endothelial cell dysfunction as a novel therapeutic target in atherosclerosis. Expert Rev Cardiovasc Ther 14:1021-1033. doi: 10.1080/14779072.2016.1207527

2. Gimbrone MA Jr, Garcia-Cardena G (2016) Endothelial Cell Dysfunction and the Pathobiology of Atherosclerosis. Circ Res 118:620-636. doi: 10.1161/circresaha.115.306301

3. DTN H and KS H (2019) Therapeutic targets for endothelial dysfunction in vascular diseases. Arch Pharm Res 42:848-861. doi: 10.1007/s12272-019-01180-7

4. Bijlsma MF, Peppelenbosch MP, Spek CA (2006) Hedgehog morphogen in cardiovascular disease. Circulation 114:1985-1991. doi: 10.1161/circulationaha.106.619213

5. C PHDNMW, Y J K, J L and A S (2016) Cellular Cholesterol Directly Activates Smoothened in Hedgehog Signaling. Cell 166:1176-1187e14. doi: 10.1016/j.cell.2016.08.003

6. Hall ET, Cleverdon ER, Ogden SK (2019) Dispatching Sonic Hedgehog: Molecular Mechanisms Controlling Deployment. Trends Cell Biol 29:385-395. doi: 10.1016/j.tcb.2019.02.005 
7. MF B, CA S and MP P (2006) Hedgehog turns lipoproteins into Janus-faced particles. Trends Cardiovasc Med 16:217-220. doi: 10.1016/j.tcm.2006.04.002

8. Akhmedov A, Sawamura T, Chen C, Kraler S, Vdovenko D, Lüscher T (2021) Lectin-like oxidized lowdensity lipoprotein receptor-1 (LOX-1): a crucial driver of atherosclerotic cardiovascular disease. Eur Heart J 42:1797-1807. doi: 10.1093/eurheartj/ehaa770

9. Li X, Gao M, Zhu S, Yin L, Zhang B, Qi Y, Zhao Y, Yu Y, Xu L (2021) viaHengshun Aromatic Vinegar Ameliorates Vascular Endothelial Injury Regulating PKCZ-Mediated Oxidative Stress and Apoptosis. Frontiers in nutrition 8:635232. doi: 10.3389/fnut.2021.635232

10. Engin A (2017) Endothelial Dysfunction in Obesity. Advances in experimental medicine and biology 960:345-379.. doi: 10.1007/978-3-319-48382-5_15

11. A P-T SB, L H-B CM, K B-D RL, AE M (2015) Global Overview of the Epidemiology of Atherosclerotic Cardiovascular Disease. Arch Med Res 46:328-338. doi: 10.1016/j.arcmed.2015.06.006

12. Pircher A, Treps L, Bodrug N, Carmeliet $P$ (2016) Endothelial cell metabolism: A novel player in atherosclerosis? Basic principles and therapeutic opportunities. Atherosclerosis 253:247-257. doi: 10.1016/j.atherosclerosis.2016.08.011

13. Patel S, Tomar S, Sharma D, Mahindroo N, Udayabanu M (2017) Targeting sonic hedgehog signaling in neurological disorders. Neurosci Biobehav Rev 74:76-97. doi: 10.1016/j.neubiorev.2017.01.008

14. Dai R, Zhu S, Xia Y, Mao L, Mei Y, Yao Y, Xue Y, Hu B (2011) Sonic hedgehog protects cortical neurons against oxidative stress. Neurochem Res 36:67-75. doi: 10.1007/s11064-010-0264-6

15. Ghanizadeh A (2012) Malondialdehyde, Bcl-2, superoxide dismutase and glutathione peroxidase may mediate the association of sonic hedgehog protein and oxidative stress in autism. Neurochem Res 37:899-901. doi: 10.1007/s11064-011-0667-z

\section{Figures}


A

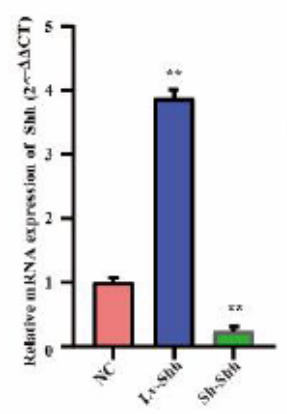

$\mathrm{C}$

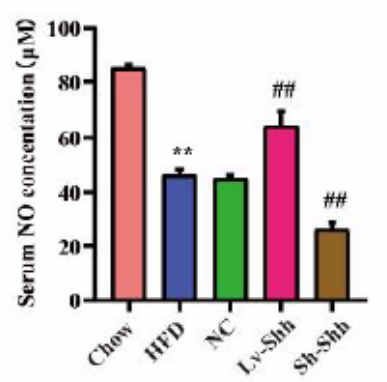

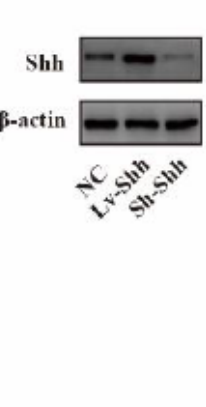
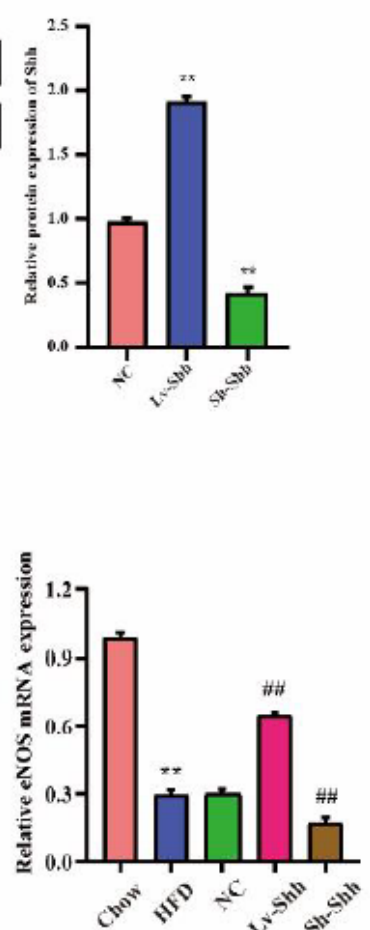

B

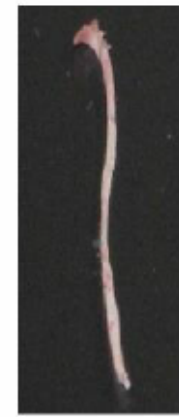

Chow

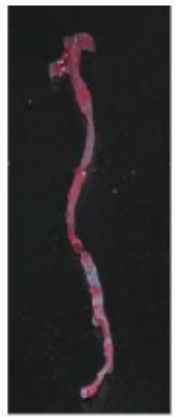

HFD

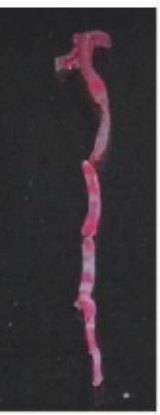

NC

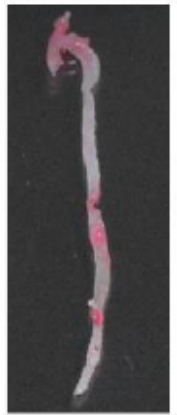

Lv-Shh

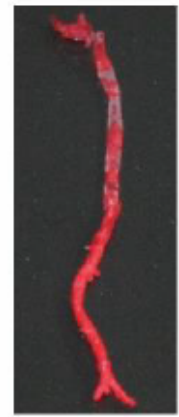

Sh-Shh
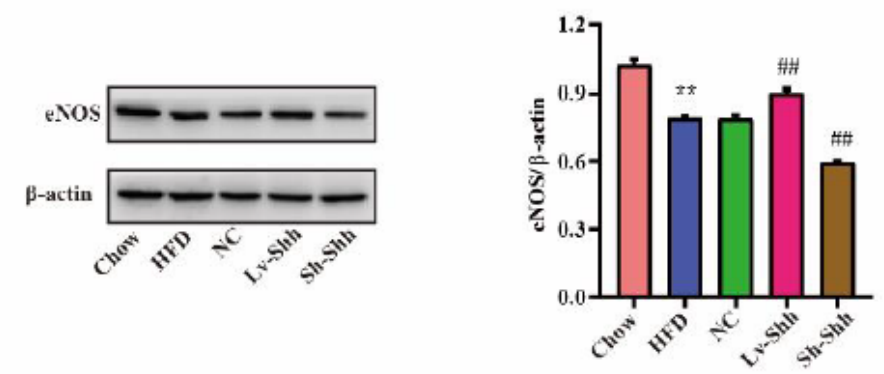

\section{Figure 1}

Effect of Shh in ApoE-/- mice in the atherosclerosis (A) Lentivirus was transfer in aortic tissue successfully. (B) aorta oil red O staining. (C) Effects of Shh intervention on NO and eNOS expression. The

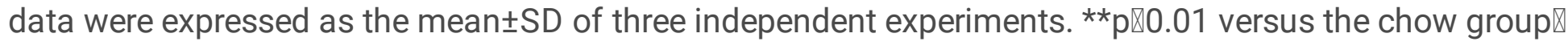
\#\#p『0.01 versus the NC group. NC: Lentivirus control group. LV-Shh: lentivirus-Shh. Sh-Shh: Shh shRNA. 
A

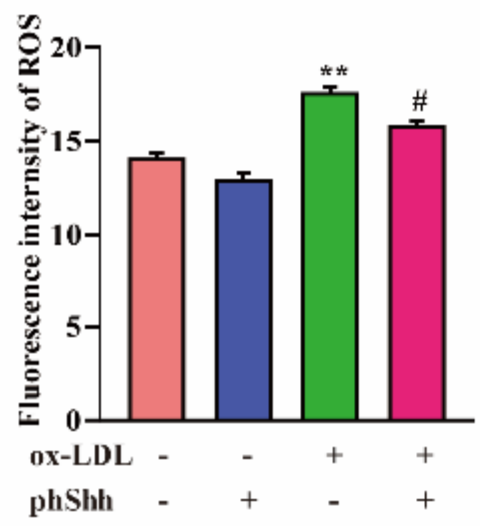

B

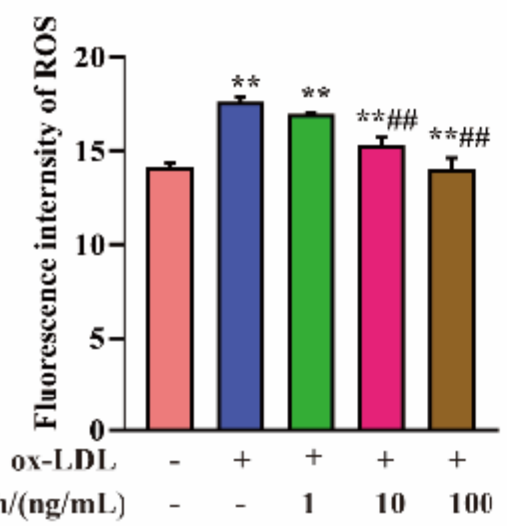

C

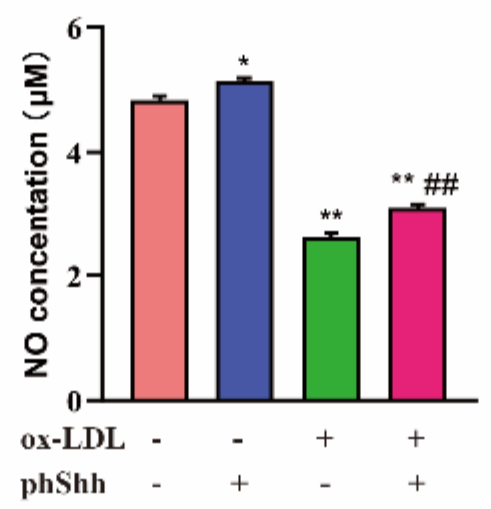

D

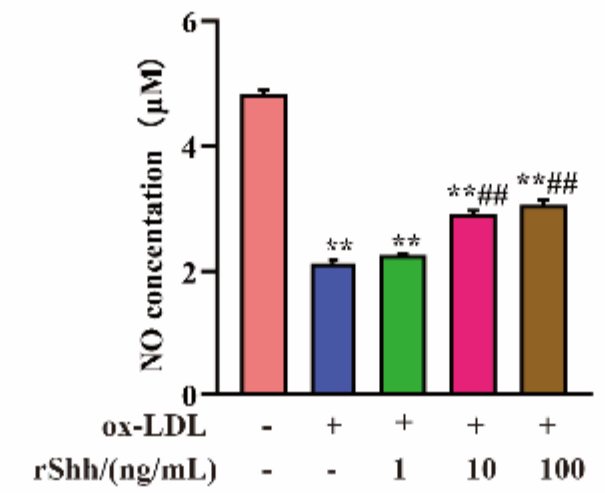

E

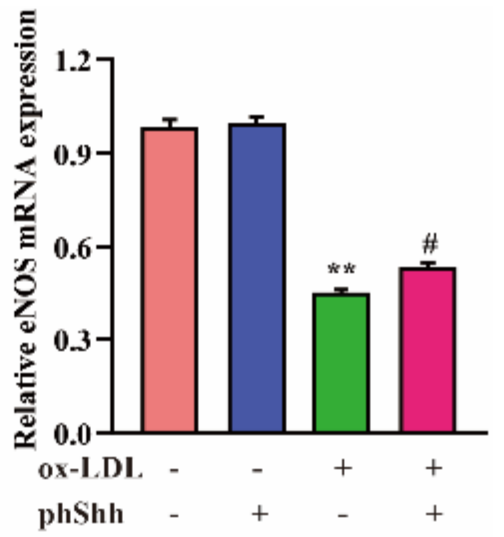

I

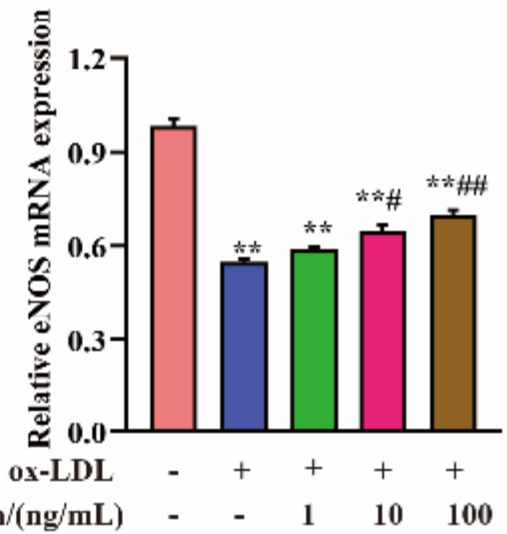

Figure 2

Effect of Shh on endothelial cell dysfunction in ox-LDL-Induced HUVECs HUVECs were transfected with plasmid encoding Shh gene for $48 \mathrm{~h}$, or pretreated with recombinant Shh protein for $24 \mathrm{~h}$, followed by coincubation with ox-LDL (50 $\mu \mathrm{g} / \mathrm{mL})$ for $24 \mathrm{~h}$. (A-B) ROS was detected by ROS assay kit. (C-D) NO was detected by Griess Reagent. (E-F) RT-qPCR was used to detect the eNOS mRNA levels. The data were expressed as the mean $\pm S D$ of three independent experiments. ${ }^{*} p<0.01$ versus the control group; \#p<0.05 and \#\#p<0.01 versus the ox-LDL treatment group. PhShh: plasmid encoding Shh gene. rShh: recombined Shh protein. 
A
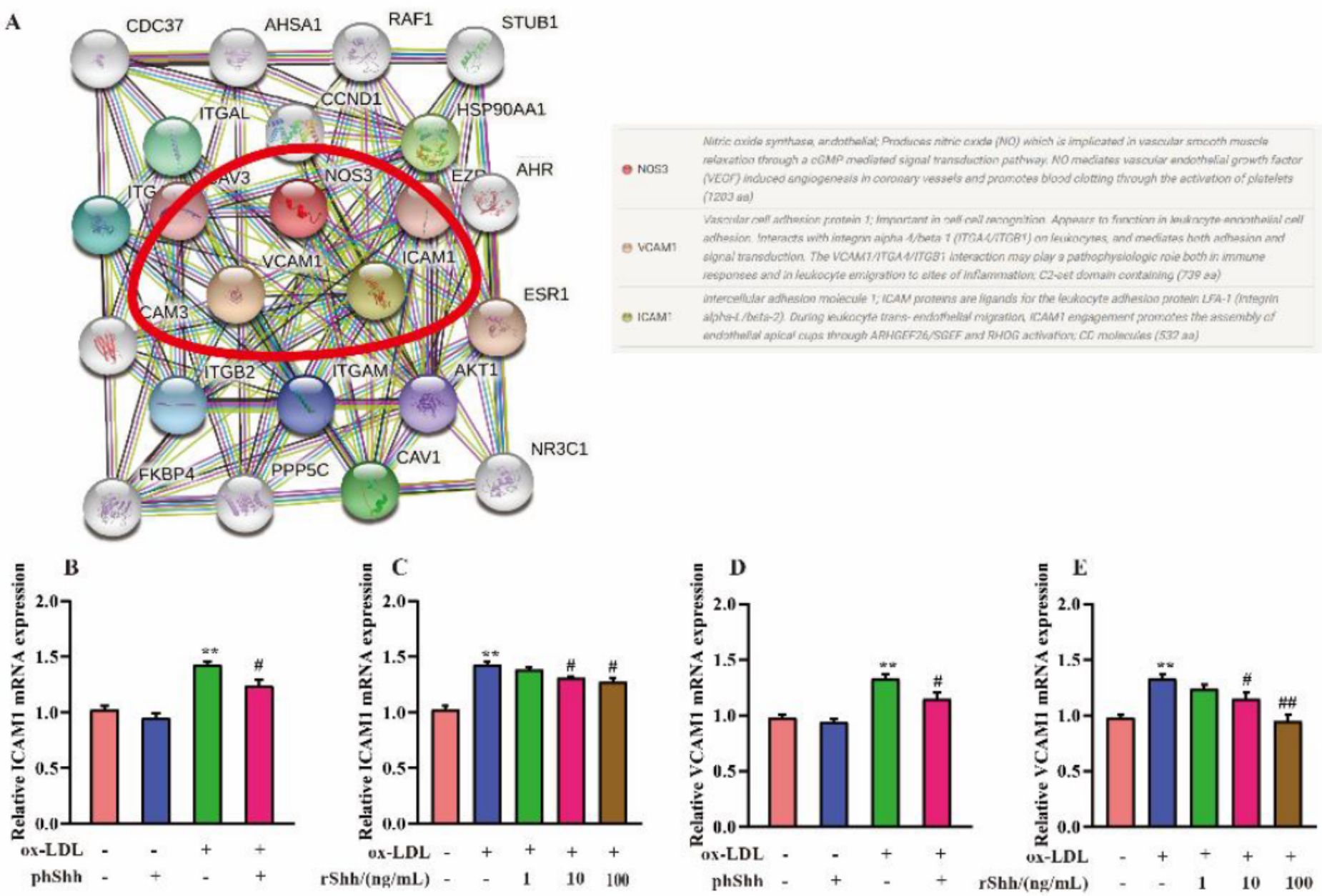

Figure 3

Effect of Shh on inflammation in ox-LDL-Induced HUVECs HUVECs were transfected with plasmid encoding Shh gene for $48 \mathrm{~h}$, or pretreated with recombinant Shh protein for $24 \mathrm{~h}$, followed by coincubation with ox-LDL $(50 \mu \mathrm{g} / \mathrm{mL})$ for $24 \mathrm{~h}$. (A) Protein-protein interaction in STRING network. (B-C) RTqPCR was used to detect the ICAM-1mRNA levels. (D-E) RT-qPCR was used to detect the ICAM-1 and VCAM-1 mRNA levels. The data were expressed as the mean \pm SD of three independent experiments. ${ }^{* *} p<0.01$ versus the control group; $\# p<0.05$ and $\# \# p<0.01$ versus the ox-LDL treatment group. PhShh: plasmid encoding Shh gene. rShh: recombined Shh protein. 

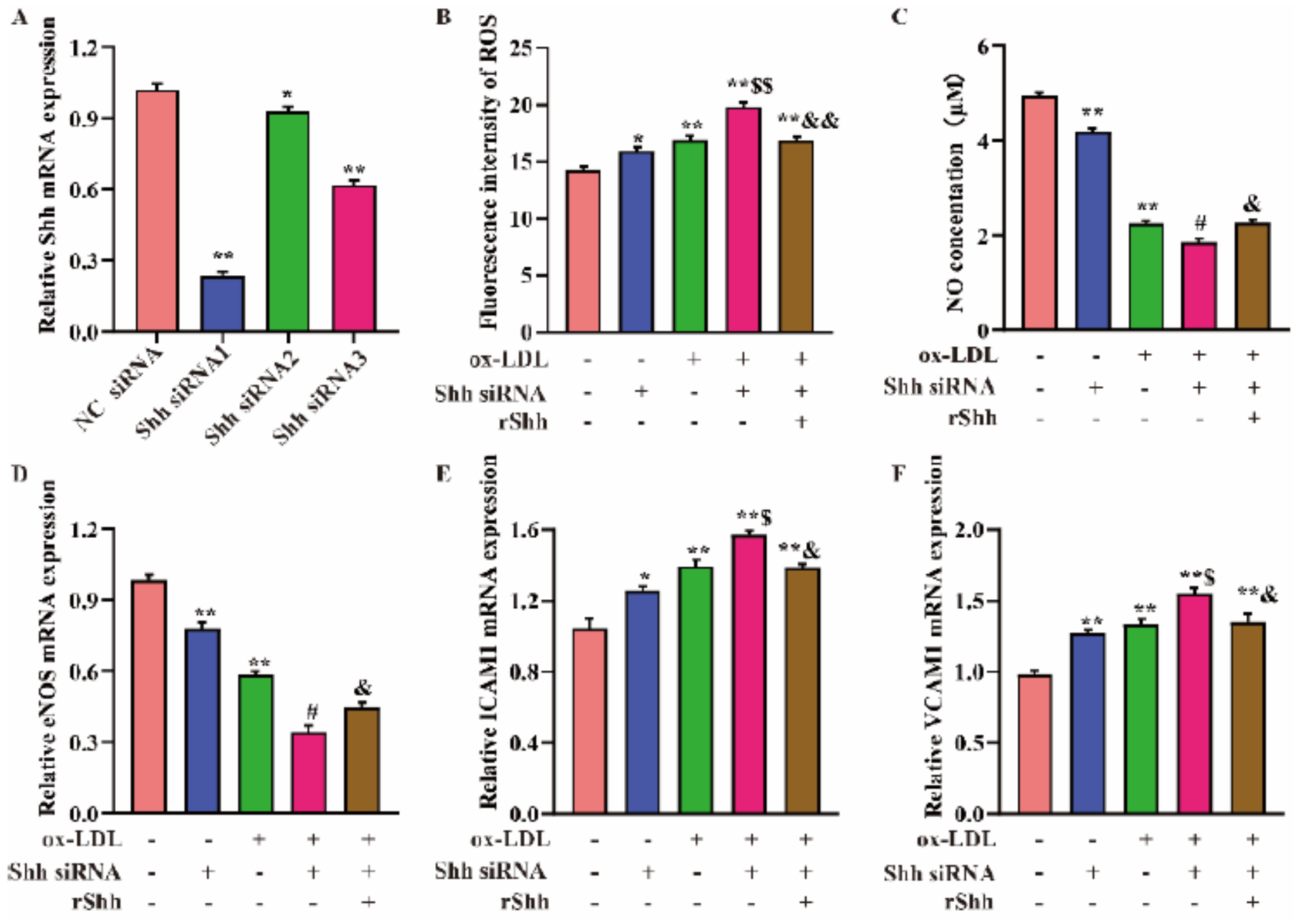

Figure 4

Silencing Shh induced endothelial cell dysfunction and inflammation HUVECs were transfected with Shh siRNA for $48 \mathrm{~h}$, followed by co-incubation with ox-LDL $(50 \mu \mathrm{g} / \mathrm{mL})$ for $24 \mathrm{~h}$. (A) Shh siRNA 1, 2, 3 could interfere Shh mRNA expression. (B)ROS was detected by ROS assay kit. (C) NO was detected by Griess Reagent. (D) RT-qPCR was used to detect the eNOS mRNA levels. (E) RT-qPCR was used to detect ICAM-1 mRAN level. (F) RT-qPCR was used to detect VCAM-1 mRNA level. The data were expressed as the mean $\pm S D$ of three independent experiments. ${ }^{* \star} p<0.01$ versus control group, $\# p<0.05$ and $\# \# p<0.01$ versus ox-LDL treatment group. \&\&p<0.01 versus rShh group. \& $p<0.05$ versus rShh group PhShh: plasmid encoding Shh gene. rShh: recombined Shh protein. 